\title{
Hajdu-Cheney Syndrome
}

National Cancer Institute

\section{Source}

National Cancer Institute. Hajdu-Cheney Syndrome. NCI Thesaurus. Code C84745.

A very rare inherited connective tissue disorder characterized by osteoporosis, skull deformities, short stature, and bone flexibility. 